\title{
A distribuição de indivíduos qualificados nas regiões metropolitanas brasileiras: \\ a influência do entretenimento e da diversidade populacional
}

André Braz Golgher Professor do Cedeplar/UFMG

\section{Palauras-chave}

qualificação, entretenimento, gays, desenvolvimento regional.

$\begin{array}{ll}\text { Classificação JEL } & \text { R23, J11, } \\ & \text { J } 60 .\end{array}$

Key words

skills, bohemia, gays, regional development.

JEL Classification R23, J11, J60.

\section{Resumo}

A base teórica do trabalho é a discussão proposta por Florida, em particular duas das hipóteses do autor que são: a importância de uma sociedade local vibrante na atração de talentos, e a importância de uma sociedade tolerante como fator de aumento da atratividade local. Foi feita uma análise empírica sobre a distribuição de indivíduos qualificados nas regiões metropolitanas do Brasil (RMs). Foram analisadas empiricamente as hipóteses desse autor mediante regressões múltiplas em painel. Verificou-se que uma parte do que foi proposto por Florida é corroborado empiricamente: aquelas que tinham índices de entretenimento, proxi para a primeira das hipóteses citadas acima, mais elevados tendiam a apresentar maiores proporções de indivíduos qualificados. Esse fato não foi observado para os índices de diversidade populacional, proxi para a segunda das hipóteses, que tiveram resultados não significativos. Por fim, as RMs foram classificadas com a utilização da técnica multivariada de aglomerados em grupos similares.

\section{Abstract:}

This paper is based theoretically on the discussion presented by Florida, in particular two of his bypotheses. The first one states that the concentration of bohemians in an area signals an environment that attracts talented people. The second emphasizes that more tolerant societies are also more attractive to creative people. Firstly, I analyzed the distribution of skilled individuals in all metropolitan regions in Brazil. Moreover, I studied empirically these two bypotheses with the use of panel analyses. Levels of Bohemia, which were used as a proxi for the first hypothesis, were positively correlated with the proportion of skilled individuals in the locality. However, I did not observe any significant correlation between population diversity, the proxy for the second bypothesis, and local levels of skills. Lastly, I classified the metropolitan regions in Brazil with the use of cluster analyses in similar groups. 


\section{Introdução}

O desenvolvimento regional pode ser analisado com base em diferentes perspectivas (Audretsch e Feldman, 1996; Simon, 1998; Feldman, 2000; Glaeser, 2000; Neary, 2001 e Scott, 2004), e um fator determinante é a distribuição espacial de capital humano (Florida, 2002a e 2005; Glaeser et al., 1995 e 2001). Segundo Lucas (1988) e Simon (1998), o ponto-chave para o crescimento e desenvolvimento de cidades e regiões é o aumento na produtividade associado à aglomeração de capital humano ou de pessoas qualificadas. Seguindo essa perspectiva, a acumulação de capital humano é a melhor estratégia de desenvolvimento regional no longo prazo (Mathur, 1999).

Entretanto, segundo o ponto de vista da teoria neoclássica (Clark et al., 2000), a atratividade regional é parcialmente causada pelas diferenças regionais de oferta e demanda de trabalhadores. A formação e a mobilidade de trabalhadores impactam na razão regional de capital/trabalho, de forma que uma região inicialmente heterogênea segundo os valores dessa razão apresentaria tendência de homogeneização dessas (Evans, 1990; Harrigan e McGregor, 1993; Graves e Mueser, 1993; Schachter e Althaus, 1993). Em muitas áreas, porém, não foi obser- vada evidência empírica significativa de convergência dos níveis espaciais de capital/trabalho, como esperado por essa teoria (Lucas, 1988; Kanbur e Rapoport, 2005). Ou seja, a concentração de indivíduos qualificados em determinadas localidades apresentou características não totalmente antecipadas por esses modelos de equilíbrio regional, uma vez que indivíduos mais qualificados tendem a se concentrar em certas localidades (Acemoglu, 1996).

Quais são os impactos da concentração de indivíduos qualificados no desenvolvimento regional? Como discute Acemoglu (1996), os retornos sociais da educação são superiores aos retornos privados, visto que interações entre indivíduos promovem transbordamento de habilidades por causa do nível individual mais elevado de capital humano com aumento na eficiência da economia e de instituições. Moretti (2004) estimou esse transbordamento e observou que a produtividade de indivíduos qualificados e também dos não qualificados aumenta como uma maior proporção de pessoas qualificadas em uma dada região. Em uma perspectiva similar, como discutido por Mathur (1999), o acúmulo de capital humano tem externalidades positivas que promovem o desenvolvimento eco- 
nômico, com incremento da produtividade do trabalho e do capital.

Segundo Audresth e Feldman (1996), como a transmissão do transbordamento associado ao acúmulo de capital humano apresenta relação decrescente com a distância graças aos custos de transmissão, indústrias e pessoas tendem a se concentrar em determinadas localidades, especialmente em atividades com grande utilização de capital humano. Deste modo, de forma geral, cidades com maior densidade de indivíduos qualificados detêm padrão de crescimento mais dinâmico (Feldman, 2000 e Glaeser, 2000), com a concentração de pessoas criativas (Jacobs, 2001). Seguindo essa perspectiva, atividades relacionadas às artes e à ciência dependem da interação entre diversos atores e têm como lócus preferencial a cidade (Lucas, 1988). Assim, produção e inovação devem ser discutidas valendo-se de um contexto geográfico bem definido (Audresth e Feldman, 1996), como núcleos urbanos (Lucas, 1988).

Alguns desses núcleos urbanos tendem a concentrar indivíduos qualificados por causa de aspectos regionais específicos, que promovem maior atratividade relativa ante as demais localidades (Stillwell e Congdon, 1991). Os fatores econômicos são em geral considerados os mais importantes na atração e na retenção de indivíduos. Apesar desse predomínio, variáveis não econômicas também são importantes, principalmente para as camadas de maior renda e escolaridade (Greenwood, 1985; Knapp e Graves, 1989 e Porrel, 1982). Além disso, essas variáveis não pecuniárias apresentam importância cada vez maior na atratividade relativa regional (Florida, 2002b; Glaeser et al., 2001 e De Hann, 1999).

Florida (2002a e 2002b) apresenta dois outros fatores que também seriam decisivos na atratividade relativa espacial. O primeiro dos fatores que impactaria positivamente na atratividade local seria a existência de uma sociedade local vibrante, o que criaria uma atmosfera favorável à atração de indivíduos talentosos e criativos. Locais criativos podem ser caracterizados como áreas com alto grau de interação em escala humana, na linha discutida por Jacobs (2001) e Wojan et al. (2007). Florida (2002a e 2002b) utilizou como proxi para uma sociedade vibrante a presença de um grande contingente de pessoas que trabalham em atividades relacionadas ao entretenimento. Indivíduos nesse tipo de atividade têm como ferramenta fundamental de trabalho a própria criatividade e também 
tendem a ser mais móveis que os demais trabalhadores, uma vez que são, em sua maioria, trabalhadores por conta própria (Wojan et al., 2007).

Segundo Florida (2002a e 2002b), outro fator que influenciaria positivamente na atratividade regional seria a diversidade populacional existente em uma localidade, que refletiria em maior tolerância na absorção de indivíduos de diferentes tipos, inclusive os muito talentosos e criativos. Como proxi para a diversidade populacional, o autor utilizou a proporção local de homossexuais na população.

Essas duas hipóteses de Florida já foram testadas para diferentes regiões. Wojan et al. (2007) analisaram counties nos EUA continental e testaram empiricamente a primeira das hipóteses. Esses verificaram que artistas e outros trabalhadores do setor criativo tendem a ser atraídos pelas mesmas características locais não observáveis. Assim, esses fatores que atraem indivíduos em ocupações relacionadas ao entretenimento também impactam positivamente na dinâmica econômica. Ainda com relação à primeira das hipóteses, Boschma e Fritsch (2007) examinaram alguns países europeus e observaram um impacto positivo entre a proporção de indivíduos em atividades de entretenimento e a proporção de empregos criativos na economia local. Além desses autores, Falck et al. (2009) estudaram essa mesma relação para dados da Alemanha e também verificaram correlação positiva e significativa entre entretenimento e estoque de capital humano.

No que concerne à segunda das hipóteses de Florida (2002a e 2002b), Westlund e Caldoni-Lundberg (2007) analisaram se sociedades civis heterogêneas e com maior tolerância influenciariam o crescimento regional de forma positiva. Os autores estudaram prefeituras no Japão e não encontraram nenhuma evidência significativa. Florida e coautores também testaram empiricamente a hipótese em diferentes contextos. Lee, Florida e Acs (2004) observaram a relação entre a tolerância e a formação de novas firmas em regiões metropolitanas nos EUA e verificaram uma associação significativa e positiva com a criação de indústrias na área de serviços. Mellander e Florida (2007) analisaram diferentes regiões da Suécia e verificaram que, além da presença de universidades, amenidades e diversidade de serviços, maior tolerância afetava positivamente a distribuição de capital humano. Utilizando uma metodologia similar a este último trabalho, 
Florida, Mellander e Stolarick (2007) observaram ainda a influência positiva da tolerância na distribuição de capital humano nas regiões metropolitanas dos EUA. Ou seja, segundo esses autores, parece existir relação, mesma que pequena, entre tolerância e distribuição de capital humano.

Especificamente para o Brasil, Golgher (2008) analisou empiricamente a primeira das hipóteses de Florida (2002a e 2002b) para dados de municípios em 2000. Uma vez controlados os efeitos relacionados à população, urbanização, localização geográfica e distribuição da economia em setores, o autor não constatou correlação positiva entre a proporção de pessoas que trabalham em posições relacionadas ao entretenimento e a qualificação média local.

O presente texto tem como objetivos centrais fazer uma análise empírica sobre a distribuição de indivíduos qualificados nas regiões metropolitanas brasileiras e, principalmente, testar para o caso brasileiro as duas hipóteses de Florida simultaneamente, complementando, assim, o discutido por Golgher (2008), que analisou apenas a primeira delas. A segunda hipótese de Florida (2002a e 2002b), que utiliza como proxi o indicador relacionado à diversidade populacional ou população gay, é preferencialmente analisada em regiões com grande população por causa de problemas referentes ao tamanho da amostra. Isso justifica a inclusão desse tipo de análise apenas para RMs e não para áreas com população menor, como municípios. Assim, este trabalho parte de um arcabouço teórico similar ao discutido por Golgher (2008), mas, de forma complementar a este, aqui são analisadas as duas hipóteses de Florida em conjunto. Além disso, inclui-se uma análise temporal com dados de 1991 e 2000 e utiliza-se uma técnica econométrica distinta, com dados em painel.

Para tanto, o texto foi dividido em sete seções, sendo a primeira delas esta introdução. Na segunda, é apresentada uma breve discussão sobre o contexto sociodemográfico das regiões metropolitanas brasileiras. Na seção seguinte, são analisados diversos indicadores de qualificação, muitos deles baseados na metodologia utilizada em Florida (2002a, 2002b e 2005). Esses indicadores foram confeccionados com a utilização dos microdados dos Censos Demográficos do Brasil de 1991 e de 2000 e são: a proporção de trabalhadores no setor criativo, o índice de qualificação superior, o índice de qualificação de pós-graduação, o índice de qualificação técnica superior, o 
índice de qualificação técnica de pósgraduação, e um índice síntese. É assim traçado um panorama da distribuição de pessoas qualificadas no país. $\mathrm{Na}$ quarta seção, com o intuito de se testar as duas hipóteses propostas por Florida citadas acima, foram estimados o índice de entretenimento e os índices gay relativo, absoluto e composto. Em seguida, são apresentadas as análises econométricas com dados em painel, que discutem empiricamente as hipóteses de Florida para os dados brasileiros referentes às RMs. $\mathrm{Na}$ sexta parte do texto, as regiões metropolitanas foram analisadas com base na técnica de aglomerados e foram classificadas segundo o perfil de cada uma delas em tópicos como qualificação e índice de entretenimento. A seção final conclui o trabalho.

\section{2_ Contexto sociodemográfico das regióes metropolitanas brasileiras}

Esta seção discute alguns pontos que servirão de contexto para a apresentação subsequente com os indicadores de qualificação. São discutidos pontos relacionados à população, à escolaridade média e à renda domiciliar para cada uma das RMs, como definido no Censo Demográfico brasileiro de 2000, incluindo os entornos e as áreas de expansão. Essas são: Belém (PA), Grande São Luís (MA), Fortaleza (CE), Natal (RN), Recife (PE), Maceió (AL), Salvador (BA), Belo Horizonte (MG), Vale do Aço (MG), Grande Vitória (ES), Rio de Janeiro (RJ), São Paulo (SP), Baixada Santista (SP), Campinas (SP), Curitiba (PR), Londrina (PR), Maringá (PR), Florianópolis (SC), Núcleo Metropolitano do Vale do Itajaí (SC), Norte/Nordeste Catarinense (SC), Porto Alegre (RS), Goiânia (GO) e Brasília (DF). Além dessas áreas, foi incluído na análise o município de Manaus (AM) (o qual, por simplicidade, será denominado de RM no restante do artigo), também um centro urbano populoso, apesar de não ser uma região metropolitana. Os dados de 1991 foram compatibilizados para que abrangessem as mesmas áreas definidas em 2000.

Inicialmente, deve-se enfatizar que existem vantagens em discutir dados de qualificação para RMs, em vez de se usar dados municipais como apresentado em Golgher (2008), já que existe importante dinâmica intraurbana de pendulariedade de indivíduos qualificados entre os diferentes municípios de uma mesma região metropolitana. Assim, como discutido em Glaeser (2000), os indivíduos analisados vivem e trabalham em sua maioria na 
mesma localidade, o que não ocorreria se municípios metropolitanos fossem discutidos em separado. Portanto, acredita-se que o estudo com regiões metropolitanas tende a ser mais relevante em vários aspectos relacionados ao desenvolvimento regional, uma vez que existe transbordamento entre os municípios de uma mesma região metropolitana (Simon, 1998).

A Tabela 1 apresenta a população, a escolaridade média da população em idade ativa, aqui definida como de 15 a 64 anos, e a renda domiciliar per capita de cada uma das RMs em 1991 e em 2000. Note que a RM de menor população nos dois anos estudados era Maringá (PR), com quase 500 mil habitantes no fim do período. No outro extremo, apareciam São Paulo (SP) e o Rio de Janeiro $(\mathrm{RJ})$, com mais de 10 milhões de habitantes em 2000. Ou seja, são todos eles centros urbanos relativamente populosos ou grandes metrópoles. A maioria das RMs apresentou crescimento populacional, com uma única exceção, o Vale do Aço (MG), que mostrou pequeno declínio em seu número de habitantes.

A escolaridade média da população em idade ativa apresentou incremento em todas as RMs, mostrando que houve um aumento médio geral da qualificação no Brasil, no período analisado.
Os valores variavam em 1991 entre 5,0 anos de estudo no Vale do Aço (MG) e 7,2 em Florianópolis (SC) e no Rio de Janeiro (RJ), e entre 6,3 em Maceió (AL) e 8,0 nestas mesmas duas últimas RMs no fim do período. Foi também observado avanço com relação à renda domiciliar per capita, com um aumento entre 1991 e 2000 em todas as RMs, com uma única exceção, Manaus(AM).

As últimas duas linhas da Tabela 1 exibem a tendência temporal de cada uma das variáveis. Para tanto, para cada uma das variáveis em separado, foi estimado o coeficiente de Pearson entre os valores da variável em 1991 e sua variação entre 1991 e 2000. Valores negativos e significativos indicam tendência de convergência, e o contrário ocorre para valores positivos e significativos. Note que não foi observada correlação significativa entre as taxas de crescimento populacional e os valores de população em 1991, indicando que não houve tendência significativa de convergência ou divergência na população das RMs. Com relação aos níveis educacionais, foi observado um coeficiente de correlação negativo e significativo $(-0,54)$, indicando tendência de homogeneização nas RMs brasileiras. Entretanto, ao contrário do verificado para escolaridade, foi observa- 
Tabela 1_Dados sociodemográficos das regióes metropolitanas no Brasil, em 1991 e 2000

\begin{tabular}{|c|c|c|c|c|c|c|}
\hline \multirow[t]{2}{*}{ Região Metropolitana } & \multicolumn{2}{|c|}{ População } & \multicolumn{2}{|c|}{$\begin{array}{l}\text { Escolaridade média das } \\
\text { pessoas em idade ativa } \\
\text { (anos de estudo) }\end{array}$} & \multicolumn{2}{|c|}{$\begin{array}{l}\text { Renda domiciliar } \\
\text { "per capita" média } \\
\text { (salários mínimos) }\end{array}$} \\
\hline & 1991 & 2000 & 1991 & 2000 & 1991 & 2000 \\
\hline Baixada Santista (SP) & 1.220 .249 & 1.476 .820 & 6,7 & 7,7 & 2,9 & 3,5 \\
\hline Belém (PA) & 1.401 .305 & 1.795 .536 & 6,8 & 7,6 & 2,1 & 2,1 \\
\hline Belo Horizonte (MG) & 3.910 .107 & 4.819 .739 & 6,4 & 7,4 & 2,4 & 3,1 \\
\hline Campinas (SP) & 1.798 .646 & 2.338 .148 & 6,4 & 7,6 & 3,2 & 3,7 \\
\hline Curitiba $(\mathrm{PR})$ & 2.061 .531 & 2.726 .581 & 6,7 & 7,8 & 2,8 & 3,7 \\
\hline Distrito Federal (DF) & 2.161 .709 & 2.952 .564 & 6,8 & 7,6 & 3,2 & 3,9 \\
\hline Florianópolis (SC) & 629.053 & 816.419 & 7,2 & 8,0 & 2,7 & 3,9 \\
\hline Fortaleza (CE) & 2.401 .537 & 2.984 .689 & 5,7 & 6,8 & 1,6 & 2,0 \\
\hline Goiânia (GO) & 1.171 .463 & 1.639 .516 & 6,8 & 7,6 & 2,4 & 3,1 \\
\hline Londrina (PR) & 553.314 & 647.854 & 6,2 & 7,6 & 2,3 & 3,0 \\
\hline Maceió (AL) & 786.488 & 989.182 & 5,6 & 6,3 & 1,7 & 2,0 \\
\hline Manaus (AM) & 1.011 .501 & 1.405 .835 & 6,5 & 7,4 & 2,4 & 2,2 \\
\hline Maringá (PR) & 381.569 & 474.202 & 6,1 & 7,5 & 2,1 & 2,7 \\
\hline Natal (RN) & 826.208 & 1.043 .321 & 6,1 & 7,1 & 1,8 & 2,3 \\
\hline Norte/Nordeste Catarinense (SC) & 712.501 & 906.982 & 6,3 & 7,3 & 2,5 & 2,7 \\
\hline Porto Alegre (RS) & 3.333 .358 & 3.658 .376 & 6,9 & 7,9 & 2,8 & 3,7 \\
\hline Recife (PE) & 2.919 .979 & 3.337 .565 & 6,3 & 7,2 & 1,8 & 2,2 \\
\hline Rio de Janeiro (RJ) & 9.814 .574 & 10.894 .156 & 7,2 & 8,0 & 2,7 & 3,7 \\
\hline Salvador $(\mathrm{BA})$ & 2.496 .521 & 3.021 .572 & 6,6 & 7,6 & 2,2 & 2,5 \\
\hline São Luís (MA) & 820.137 & 1.070 .688 & 6,9 & 7,8 & 1,5 & 1,8 \\
\hline São Paulo (SP) & 15.444 .941 & 17.879 .997 & 6,7 & 7,9 & 3,6 & 4,0 \\
\hline Vale do Aço (MG) & 602.148 & 563.258 & 5,0 & 6,6 & 1,4 & 1,8 \\
\hline Vale do Itajaí (SC) & 451.713 & 558.165 & 6,3 & 7,2 & 2,5 & 3,1 \\
\hline Vitória (ES) & 1.126 .638 & 1.425 .587 & 6,5 & 7,6 & 2,2 & 2,9 \\
\hline $\begin{array}{l}\text { Coeficiente de Pearson entre os va- } \\
\text { lores da variável em } 1991 \text { e sua va- } \\
\text { riação entre } 1991 \text { e } 2000\end{array}$ & \multicolumn{2}{|c|}{$-0,29$} & \multicolumn{2}{|c|}{$-0,54 * * *$} & \multicolumn{2}{|c|}{$0,35^{*}$} \\
\hline Tendência & \multicolumn{2}{|c|}{ Não significativa } & \multicolumn{2}{|c|}{ Convergência } & \multicolumn{2}{|c|}{ Divergência } \\
\hline
\end{tabular}


da tendência significativa em $10 \%$ de divergência nos valores de renda per capita.

\section{Análise dos indicadores de qualificação}

Neste item, são apresentadas discussões mais detalhadas sobre qualificação com os indicadores mencionados acima: proporção de trabalhadores no setor criativo, índice de qualificação superior, índice de qualificação de pós-graduação, índice de qualificação técnica superior, índice de qualificação técnica de pós-graduação e índice síntese. Note que esses indicadores são os mesmos utilizados em Golgher (2008), em que são apresentados em maior detalhamento. Segue uma breve descrição do primeiro desses.

\section{Proporção de trabalhadores no setor criativo}

Esse indicador é similar ao utilizado por Florida (2005) e adaptado para os dados brasileiros com base na classificação brasileira de ocupações (FIBGE, 2000). Foram consideradas ocupações criativas as seguintes, entre elas: membros superiores do poder público, dirigentes de organizações de interesse público e de empresas e gerentes; e profissionais das ciências e das artes. As demais foram consideradas não criativas. Em seguida, foi feita a compatibilização entre os Censos.
Sabe-se que o termo "setor criativo" como utilizado neste texto apresenta limitações e imperfeições. Entretanto, para que uma comparabilidade fosse possível com relação aos trabalhos de Florida (2002a, 2002b e 2005), optou-se em utilizar a mesma definição e nomenclatura desse autor.

Como mostra Florida (2005), a economia criativa absorvia aproximadamente $30 \%$ dos trabalhadores nos EUA, e a participação desse setor na economia apresentava tendência de expansão relativa. Para a Europa, entre os países analisados, a proporção de trabalhadores na economia criativa variava entre $13 \%$, em Portugal, e 30\%, na Bélgica (Florida e Tinagli, 2004). O Brasil, por ter uma economia menos sofisticada que as citadas acima, deve apresentar proporções menores que esses países.

A Tabela 2 mostra a proporção de trabalhadores na economia criativa nas RMs brasileiras, nos anos 1991 e 2000. Os valores variavam no primeiro desses anos entre 6,5\% para o Vale do Aço (MG) e 12,8\% em Florianópolis (SC). Em 2000, a proporção variava entre $8,1 \%$ para essa primeira RM e 14,4\% para São Paulo (SP), valores inferiores ao verificado nos EUA (Florida, 2002a, 2002b e 2005) e Europa (Florida e Tinagli, 2004). Todas as 
Tabela 2_Proporção de trabalhadores na economia criativa e índices de qualificação nas regiões metropolitanas no Brasil, em 1991 e 2000

\begin{tabular}{|c|c|c|c|c|c|c|c|c|c|c|c|c|}
\hline \multirow[t]{2}{*}{ Região Metropolitana } & \multicolumn{2}{|c|}{$\begin{array}{c}\text { Proporção de } \\
\text { trabalhadores na } \\
\text { economia criativa } \\
\text { (\%) }\end{array}$} & \multicolumn{2}{|c|}{$\begin{array}{c}\text { Índice de } \\
\text { qualificação } \\
\text { superior (\%) }\end{array}$} & \multicolumn{2}{|c|}{$\begin{array}{c}\text { Índice de } \\
\text { qualificação } \\
\text { de pós-graduação } \\
(\%)\end{array}$} & \multicolumn{2}{|c|}{$\begin{array}{c}\text { Índice técnico } \\
\text { superior } \\
\text { [\%] }\end{array}$} & \multicolumn{2}{|c|}{$\begin{array}{c}\text { Índice técnico de } \\
\text { pós-graduação } \\
(\%)\end{array}$} & \multicolumn{2}{|c|}{$\begin{array}{l}\text { Indicador } \\
\text { síntese }\end{array}$} \\
\hline & 1991 & 2000 & 1991 & 2000 & 1991 & 2000 & 1991 & 2000 & 1991 & 2000 & 1991 & 2000 \\
\hline Baixada Santista (SP) & 10,8 & 12,3 & 7,8 & 9,2 & 0,18 & 0,51 & 9,1 & 10,7 & 0,05 & 0,12 & 0,58 & 0,64 \\
\hline Belém (PA) & 10,0 & 10,3 & 5,9 & 6,6 & 0,31 & 0,66 & 7,4 & 7,8 & 0,09 & 0,13 & 0,57 & 0,54 \\
\hline Belo Horizonte (MG) & 11,7 & 12,3 & 7,5 & 8,3 & 0,37 & 0,80 & 8,4 & 9,0 & 0,08 & 0,14 & 0,64 & 0,64 \\
\hline Campinas (SP) & 11,0 & 12,8 & 8,1 & 9,5 & 0,66 & 1,22 & 8,7 & 10,0 & 0,10 & 0,23 & 0,73 & 0,79 \\
\hline Curitiba (PR) & 12,2 & 13,4 & 8,9 & 10,2 & 0,39 & 1,03 & 9,9 & 10,6 & 0,10 & 0,17 & 0,72 & 0,76 \\
\hline Distrito Federal (DF) & 11,3 & 12,7 & 9,7 & 9,8 & 0,69 & 1,24 & 10,5 & 10,2 & 0,14 & 0,17 & 0,84 & 0,76 \\
\hline Florianópolis (SC) & 12,8 & 13,6 & 9,8 & 11,2 & 1,07 & 2,04 & 10,4 & 10,0 & 0,15 & 0,25 & 0,94 & 0,92 \\
\hline Fortaleza (CE) & 8,9 & 10,0 & 5,4 & 5,7 & 0,25 & 0,61 & 6,3 & 6,5 & 0,05 & 0,11 & 0,46 & 0,48 \\
\hline Goiânia (GO) & 12,2 & 12,2 & 7,5 & 7,8 & 0,30 & 0,65 & 8,0 & 7,9 & 0,07 & 0,10 & 0,62 & 0,57 \\
\hline Londrina (PR) & 10,7 & 12,6 & 7,7 & 9,6 & 0,48 & 0,84 & 8,0 & 9,8 & 0,06 & 0,15 & 0,61 & 0,69 \\
\hline Maceió (AL) & 9,3 & 11,4 & 6,7 & 7,1 & 0,17 & 0,55 & 8,6 & 9,2 & 0,03 & 0,10 & 0,49 & 0,56 \\
\hline Manaus (AM) & 7,5 & 9,7 & 4,0 & 4,5 & 0,19 & 0,59 & 5,0 & 5,4 & 0,05 & 0,13 & 0,38 & 0,45 \\
\hline Maringá (PR) & 10,9 & 11,1 & 7,0 & 8,4 & 0,44 & 0,94 & 7,4 & 8,3 & 0,03 & 0,06 & 0,56 & 0,58 \\
\hline Natal (RN) & 10,7 & 12,4 & 6,7 & 7,3 & 0,38 & 0,77 & 8,4 & 8,7 & 0,11 & 0,14 & 0,64 & 0,62 \\
\hline $\begin{array}{l}\text { Norte/Nordeste } \\
\text { Catarinense (SC) }\end{array}$ & 8,0 & 10,1 & 4,3 & 5,6 & 0,15 & 0,51 & 4,8 & 6,0 & 0,03 & 0,11 & 0,36 & 0,47 \\
\hline Porto Alegre (RS) & 12,2 & 13,8 & 8,2 & 9,1 & 0,46 & 1,01 & 9,1 & 9,3 & 0,09 & 0,15 & 0,70 & 0,71 \\
\hline Recife (PE) & 10,8 & 12,0 & 7,4 & 7,9 & 0,29 & 0,66 & 9,4 & 10,1 & 0,08 & 0,13 & 0,63 & 0,63 \\
\hline Rio de Janeiro (RJ) & 12,2 & 14,0 & 9,7 & 10,4 & 0,62 & 1,30 & 11,6 & 11,6 & 0,18 & 0,31 & 0,91 & 0,91 \\
\hline Salvador (BA) & 10,5 & 12,0 & 6,6 & 7,0 & 0,30 & 0,68 & 8,0 & 8,4 & 0,08 & 0,14 & 0,58 & 0,59 \\
\hline São Luís (MA) & 8,6 & 9,5 & 4,6 & 5,3 & 0,15 & 0,42 & 6,1 & 6,4 & 0,06 & 0,05 & 0,43 & 0,41 \\
\hline São Paulo (SP) & 11,9 & 14,4 & 9,0 & 10,3 & 0,43 & 0,88 & 10,4 & 11,8 & 0,10 & 0,18 & 0,74 & 0,79 \\
\hline Vale do Aço (MG) & 6,5 & 8,1 & 3,1 & 4,0 & 0,15 & 0,22 & 3,8 & 5,2 & 0,04 & 0,06 & 0,30 & 0,33 \\
\hline Vale do Itajaí (SC) & 9,1 & 10,5 & 4,5 & 6,0 & 0,21 & 0,50 & 4,9 & 6,0 & 0,04 & 0,04 & 0,40 & 0,43 \\
\hline Vitória (ES) & 10,5 & 11,6 & 6,5 & 7,5 & 0,26 & 0,65 & 7,4 & 8,1 & 0,06 & 0,12 & 0,54 & 0,57 \\
\hline Total & 11,4 & 12,9 & 8,1 & 9,0 & 0,43 & 0,92 & 9,4 & 10,0 & 0,10 & 0,18 & 0,70 & 0,72 \\
\hline $\begin{array}{l}\text { Coeficiente de Pearson } \\
\text { entre os valores da variá- } \\
\text { vel em } 1991 \text { e sua variação } \\
\text { entre } 1991 \text { e } 2000\end{array}$ & \multicolumn{2}{|c|}{-026} & \multicolumn{2}{|c|}{0,10} & \multicolumn{2}{|c|}{$0,87 * * *$} & \multicolumn{2}{|c|}{$-0,31$} & \multicolumn{2}{|c|}{$0,42 * *$} & \multicolumn{2}{|c|}{$-0,44 * *$} \\
\hline $\begin{array}{l}\text { Tendência } \\
\text {............... }\end{array}$ & \multicolumn{2}{|c|}{ Não significativa } & \multicolumn{2}{|c|}{ Não significativa } & \multicolumn{2}{|c|}{ Divergência } & \multicolumn{2}{|c|}{ Não significativa } & \multicolumn{2}{|c|}{ Divergência } & \multicolumn{2}{|c|}{ Convergência } \\
\hline
\end{tabular}

* Significativa em $10 \%$; ** Significativa em $5 \%$; *** Significativa em $1 \%$.

Fonte: IBGE. Censos Demográficos (1991 e 2000). Elaboração própria. 
RMs mostraram variação positiva para o indicador entre os anos analisados, exibindo sofisticação do mercado de trabalho em todas elas.

De forma semelhante à Tabela 1, na parte inferior da Tabela 2, é mostrado o coeficiente de Pearson entre os valores em 1991 e a variação desses no período. Note que, para a proporção de trabalhadores na economia criativa, o coeficiente foi não significativo e, assim, não foi verificada nenhuma tendência de convergência ou divergência significativa nos valores.

A Tabela 2 mostra também os resultados para outros indicadores de qualificação. Entre esses, discute-se inicialmente o índice de qualificação superior e o índice de qualificação de pós-graduação. Esses indicadores mostram quão qualificada é a força de trabalho da RM sem que seja feita uma relação direta com as ocupações no mercado de trabalho. Segue a definição de ambos.

\section{Índice de qualificação superior}

Proporção de pessoas na população com idade entre 20 e 64 anos com pelo menos nível superior de escolaridade.

\section{Índice de qualificação de pós-graduação}

Proporção de não estudantes com mestrado/doutorado ou de estudantes cur- sando um desses níveis na mesma faixa etária acima.

Os valores para o índice de qualificação superior eram mais elevados em Florianópolis (SC), nos dois anos estudados, respectivamente $9,8 \%$ e $11,2 \%$, e mais baixos no Vale do Aço (MG), com os valores de 3,1\% e 4,0\%. Assim, como observado para a proporção de trabalhadores na economia criativa, também para o índice de qualificação superior houve incremento em todas as RMs, sem nenhuma tendência temporal significativa de variação nos valores.

Para o índice de qualificação de pós-graduação, os valores variavam em 1991 entre 0,15\% no Vale do Aço (MG) e 1,07\% em Florianópolis (SC), valores relativamente pequenos. Entretanto, verifica-se que todas as RMs apresentaram aumento marcante para esse índice, com valores entre $0,22 \%$ e 2,04\% para essas mesmas duas RMs em 2000. O coeficiente de correlação foi positivo e significativo, indicando tendência de divergência nos valores, ou seja, observou-se uma concentração relativa de indivíduos muito qualificados.

Os dois últimos indicadores discutidos mostraram a evolução positiva na qualificação geral da população em 
idade ativa ocorrida em todas as RMs. Entretanto, muitos dos indivíduos qualificados podem não estar exercendo uma ocupação que requeira níveis elevados de capital humano. Assim, com o intuito de suplantar parte dessas limitações, são incluídos dois outros indicadores o índice de qualificação técnica superior e o índice de qualificação técnica de pósgraduação. Esses indicadores são definidos a seguir:

\section{Índice de qualificação técnica superior}

Proporção de trabalhadores com nível superior em ocupações classificadas como profissionais: policientíficos; das ciências exatas, físicas e da engenharia; ou das ciências biológicas, da saúde e afins; e

\section{Índice de qualificação técnica de pós-graduação}

Proporção de trabalhadores com mestrado ou doutorado nas mesmas ocupações citadas acima.

Os resultados para esses dois índices também são mostrados na Tabela 2. De forma geral, os resultados relativos obtidos para esses índices são muito similares ao observado para os demais quanto à distribuição geral. Entretanto, ao contrário do verificado para os demais indicadores, observa-se que três RMs tiveram seu valor diminuído para o pri- meiro desses índices, Goiânia (GO), Rio de Janeiro (RJ) e Distrito Federal (DF). Ou seja, apesar de a qualificação da população ter aumentado, isso não ocorreu com relação à inserção de indivíduos com graduação no mercado de trabalho em posições técnicas qualificadas, indicando uma subutilização da mão de obra qualificada ou um viés de formação de indivíduos em outras áreas do conhecimento. Para os demais centros urbanos, a variação foi positiva. Não foi observada nenhuma variação significativa temporal entre 1991 e 2000.

Quanto ao índice de qualificação técnica de pós-graduação, assim como observado para o índice de qualificação de pós-graduação, as variações foram positivas e elevadas em quase todas as RMs. Entretanto, duas delas tiveram valores negativos para a variação do indicador, Vale do Itajaí (SC) e São Luís (MA), indicando que a relação entre a formação de indivíduos com alta qualificação e a ocupação de postos de trabalhos técnicos que requerem elevados níveis de capital humano ainda é precária nessas áreas. Aqui também se observou uma tendência de divergência significativa entre os valores, como observado para o índice de qualificação de pós-graduação. 
Como discutido em Golgher (2008), todos os indicadores acima apresentam correlação positiva e significativa. Para dados de RMs, os valores variavam entre 0,61 e 0,93 em 2000. Assim, como forma de síntese de todos esses resultados positivamente correlacionados, os índices foram combinados para que um único indicador síntese fosse confeccionado. Segue a definição do indicador:

\section{Indicador síntese}

Média ponderada dos demais indicadores.

Como mostra a Tabela 2, verifica-se que Florianópolis (SC) e o Rio de Janeiro (RJ) se destacam com valores muito superiores aos demais, com cifras acima de 0,9 nos dois anos estudados. Note que, ao contrário do demais indicadores, esse índice é relativo a cada ano em separado, tendo valor máximo de 1. Em todos os indicadores de qualificação apresentados, esses mesmos dois municípios apareciam em destaque. Em seguida, observa-se um grupo com cinco regiões metropolitanas com valores entre 0,70 e 0,84 para o indicador nos dois anos estudados: Campinas (SP), São Paulo (SP), Curitiba (PR) e Porto Alegre (RS) das regiões Sul/Sudeste, e o Distrito Federal (DF). Por outro lado, Fortaleza (CE), Norte/Nordeste Catarinense (SC), Vale do Itajaí (SC), Manaus (AM), São
Luís (MA) e o Vale do Aço (MG) tinham valores mais baixos, abaixo de 0,50 , sendo todas as RMs localizadas nas regiões Norte/Nordeste ou no interior das regiões Sul/Sudeste. As demais RMs, Baixada Santista (SP), Belém (PA), Belo Horizonte (MG), Goiânia (GO), Londrina (PR), Maceió (AL), Maringá (PR), Natal (RN), Recife (PE), Salvador (BA) e Vitória (ES), formavam um grupo intermediário com relação à qualificação. Verifica-se ainda que houve tendência geral de convergência nos indicadores síntese entre as RMs brasileiras.

\section{4_Análise dos indicadores de entretenimento e de diversidade}

Nesta seção, são apresentados os resultados para o índice de entretenimento e os índices gays ou de diversidade populacional. Tais indicadores são semelhantes aos propostos por Florida (2002a, 2002b e 2005), mas com adaptação e modificação para os dados brasileiros. O objetivo principal deste trabalho é utilizar esses indicadores para testar as hipóteses desse autor quanto à distribuição de indivíduos qualificados.

Segundo o autor, uma sociedade vibrante impactaria em uma atratividade regional mais elevada, o que influencia- 
ria na aglomeração de indivíduos qualificados e em maior produtividade local. O índice de entretenimento é utilizado com uma proxi para a existência de uma sociedade vibrante. Segue uma definição dessa proxi.

\section{Índice de entretenimento}

Este indicador é uma adaptação do índice de boemia de Florida (2005) para o caso brasileiro. Tal índice é a proporção de trabalhadores que eram alocados em ocupações classificadas como profissionais de espetáculos e das artes. O índice indica a razão entre o valor de um determinado local e o valor médio para todas as áreas analisadas.

Os índices de diversidade populacional, medida aqui pelos índices gays absolutos, relativos e composto, são uma proxi para a tolerância da sociedade local com relação a indivíduos que diferem do padrão considerado "normal", entre eles os homossexuais. Segundo Florida, (2002a, 2002b e 2005), sociedades mais tolerantes no que diz respeito à diversidade populacional propiciariam maior facilidade de adaptação para todos os tipos de indivíduo, atraindo, assim, ampla gama de pessoas, inclusive as mais qualificadas e criativas. Desta maneira, uma sociedade mais diversa teria maior poder de retenção e de absorção de indivíduos qualificados, e isso teria impacto positivo direto na promoção de atividades de alta tecnologia. Seguem as definições de cada um dos indicadores de diversidade.

\section{Índice de diversidade ou índice "gay" bruto}

Esse indicador é uma proxi para a população homossexual de uma localidade e também é baseado no apresentado em Florida (2005). É calculado como a proporção de domicílios compostos de somente dois homens não aparentados, cuja idade média era superior a 35 anos.

A única diferença entre o proposto por esse autor e o indicador deste texto é a inclusão do filtro de idade. O limite de idade foi imposto para que fossem excluídos muitos dos domicílios onde dois amigos heterossexuais dividem moradia, o que ocorre principalmente em idade mais jovem. O índice indica a razão do valor de determinado local com relação ao valor médio para todas as áreas analisadas.

\section{Índice de diversidade ou índice "gay" relativo}

Razão entre domicílios compostos de somente dois homens não aparentados com idade média acima de 35 anos com relação ao mesmo tipo de domicílio para todas as idades. O objetivo aqui é minimizar os efeitos regionais na composição domiciliar verificados empiricamente. 


\section{Índice de diversidade ou índice "gay" composto}

Média ponderada dos dois indicadores acima. O objetivo da confecção desse indicador é tentar minimizar as limitações dos dois anteriores, como será detalhado na discussão dos resultados.
A Tabela 3 mostra os resultados obtidos para esses quatro indicadores citados nos dois anos em estudo. Em 2000, os maiores valores para o índice de entretenimento são observados para as RMs próximas ao mar. Entre as 24 regiões metropolitanas, os seis maiores valores

Tahela 3_ Índice de entretenimento e índices de diversidade nas regióes metropolitanas no Brasil em 1991 e 2000

\begin{tabular}{|c|c|c|c|c|c|c|c|c|}
\hline \multirow{2}{*}{ Região Metropolitana } & \multicolumn{2}{|c|}{$\begin{array}{l}\text { Indice de } \\
\text { entretenimento }\end{array}$} & \multicolumn{2}{|c|}{$\begin{array}{l}\text { Índice de } \\
\text { diversidade bruto }\end{array}$} & \multicolumn{2}{|c|}{$\begin{array}{l}\text { Índice de } \\
\text { diversidade relativo }\end{array}$} & \multicolumn{2}{|c|}{$\begin{array}{l}\text { Índice de } \\
\text { diversidade composto }\end{array}$} \\
\hline & 1991 & 2000 & 1991 & 2000 & 1991 & 2000 & 1991 & 2000 \\
\hline Baixada Santista (SP) & 0,74 & 1,15 & 1,82 & 0,73 & 36,3 & 25,6 & 0,94 & 0,44 \\
\hline Belém (PA) & 0,88 & 1,05 & 0,81 & 1,29 & 22,9 & 27,5 & 0,50 & 0,64 \\
\hline Belo Horizonte (MG) & 1,27 & 1,07 & 0,69 & 0,74 & 27,4 & 28,1 & 0,52 & 0,46 \\
\hline Campinas (SP) & 0,57 & 0,67 & 0,67 & 1,12 & 20,5 & 35,9 & 0,43 & 0,65 \\
\hline Curitiba (PR) & 1,23 & 1,12 & 0,69 & 0,77 & 24,4 & 26,5 & 0,48 & 0,46 \\
\hline Distrito Federal (DF) & 0,77 & 0,67 & 1,03 & 1,31 & 21,2 & 23,6 & 0,54 & 0,61 \\
\hline Florianópolis (SC) & 0,85 & 1,12 & 0,29 & 0,35 & 10,1 & 12,2 & 0,20 & 0,21 \\
\hline Fortaleza (CE) & 0,84 & 1,14 & 0,63 & 1,53 & 22,9 & 34,4 & 0,45 & 0,77 \\
\hline Goiânia (GO) & 1,11 & 1,02 & 0,64 & 0,47 & 26,6 & 19,9 & 0,49 & 0,31 \\
\hline Londrina (PR) & 0,77 & 0,78 & 0,55 & 0,61 & 29,4 & 23,9 & 0,50 & 0,39 \\
\hline Maceió (AL) & 0,65 & 0,94 & 0,41 & 1,04 & 18,8 & 49,5 & 0,34 & 0,73 \\
\hline Manaus (AM) & 0,55 & 0,86 & 1,06 & 1,51 & 15,8 & 30,0 & 0,48 & 0,73 \\
\hline Maringá (PR) & 0,65 & 0,65 & 0,16 & 0,65 & 18,0 & 34,9 & 0,26 & 0,49 \\
\hline Natal (RN) & 0,82 & 1,15 & 1,29 & 0,98 & 33,0 & 39,2 & 0,75 & 0,63 \\
\hline Norte/Nordeste Catarinense (SC) & 0,52 & 0,75 & 0,16 & 0,51 & 15,0 & 31,4 & 0,22 & 0,42 \\
\hline Porto Alegre (RS) & 1,09 & 1,00 & 1,04 & 0,94 & 36,2 & 38,1 & 0,72 & 0,61 \\
\hline Recife (PE) & 0,91 & 1,03 & 0,48 & 0,48 & 26,4 & 35,4 & 0,45 & 0,44 \\
\hline Rio de Janeiro (RJ) & 1,31 & 1,22 & 1,45 & 1,29 & 41,5 & 40,2 & 0,90 & 0,74 \\
\hline Salvador (BA) & 0,96 & 1,21 & 1,27 & 1,02 & 28,3 & 33,6 & 0,69 & 0,60 \\
\hline São Luís (MA) & 0,79 & 0,93 & 0,96 & 1,18 & 23,8 & 30,3 & 0,55 & 0,63 \\
\hline São Paulo (SP) & 0,96 & 0,91 & 1,08 & 0,96 & 28,4 & 30,3 & 0,64 & 0,55 \\
\hline Vale do Aço (MG) & 0,40 & 0,58 & 0,44 & 0,92 & 21,0 & 63,4 & 0,37 & 0,80 \\
\hline Vale do Itajaí (SC) & 0,95 & 0,82 & 0,15 & 0,74 & 38,5 & 35,4 & 0,50 & 0,52 \\
\hline Vitória (ES) & 1,08 & 1,01 & 0,65 & 0,58 & 20,9 & 21,3 & 0,43 & 0,36 \\
\hline
\end{tabular}

Fonte: IBGE. Censos Demográficos (1991 e 2000). Elaboração própria. 
são para centros urbanos costeiros Baixada Santista (SP), Florianópolis (SC), Fortaleza (CE), Natal (RN), Rio de Janeiro (RJ) e Salvador (BA). Por outro lado, Campinas (SP), Maringá (PR) e Vale do Aço (MG), regiões sem mar, tinham valores inferiores a 0,70 nos dois anos estudados.

Qual a relação entre esse indicador e a proporção de pessoas em atividades criativas e índices de qualificação? A Tabela 4 - parte A mostra os coeficientes de correlação entre o índice de entretenimento e os índices de qualificação. Observe que os valores são positivos, mas significativos apenas para a proporção de trabalhadores na economia criativa e para o índice técnico de pós-gra- duação. Entretanto, note que parte das pessoas na economia criativa também é locada em ocupações de entretenimento, o que acarreta problemas de endogenia. Assim, criou-se outra variável que se refere aos trabalhadores na economia criativa que não eram ligados a atividades de entretenimento. Entre parênteses é mostrado o resultado não significativo para a correlação entre esta última variável e o índice de entretenimento. Ou seja, quando medidas em uma análise bivariada, diferentemente do proposto por Florida (2002a, 2002b e 2005), locais com maiores índices de entretenimento não tendiam a apresentar maior proporção de indivíduo em atividades criativas.

\section{Tabela 4_Coeficientes de correlação de Pearson entre variáveis e indicadores para dados de 2000}

\begin{tabular}{|c|c|c|}
\hline \multicolumn{3}{|c|}{ Variável } \\
\hline Parte A & Índice de entretenimento & Índice de diversidade composto \\
\hline Proporção de trabalhadores na economia criativa & $\begin{array}{l}0,39 * \\
(0,30)\end{array}$ & $-0,37 *$ \\
\hline Índice de qualificação superior & 0,24 & $-0,44 * *$ \\
\hline Índice de qualificação de pós-graduação & 0,17 & $-0,33$ \\
\hline Índice técnico superior & 0,33 & $-0,25$ \\
\hline Índice técnico de pós-graduação & $0,35^{*}$ & $-0,07$ \\
\hline Índice síntese & 0,32 & $-0,31$ \\
\hline Parte B & Índice de diversidade relativo & Índice de diversidade composto \\
\hline Índice de diversidade bruto & $0,31^{\#}$ & $0,87 * *$ \\
\hline Índice de diversidade relativo & & $\begin{array}{l}0,74 * * \\
\ldots \ldots \ldots\end{array}$ \\
\hline
\end{tabular}

\# Significativa em 14\%; $*$ Significativa em $10 \%$; ** Significativa em $5 \%$; Demais não significativas.

Fonte: IBGE. Censo Demográfico, (2000). Elaboração própria. 
Em seguida, são discutidos os índices de diversidade também mostrados na Tabela 3. Entretanto, deve-se ressaltar que esse indicador é muito pobre como medida de homossexualidade. Primeiro por ser uma proxi: dois homens não aparentados com idade média acima de 35 anos vivendo em um mesmo arranjo domiciliar que conta somente com esses indivíduos não necessariamente são homossexuais. Podem ser amigos, colegas de trabalho, etc. Além disso, o número de domicílios com esse arranjo domiciliar no Brasil é muito pequeno, o que inviabiliza o estudo em áreas com pequena população.

Com relação ao índice bruto, o Rio de Janeiro (RJ) apresentava um valor muito acima da média nos dois anos estudados. Esse fato pode não ser um indicativo fidedigno de que a população homossexual seja relativamente mais numerosa. Existe a possibilidade de esse número ter sido obtido devido a outras especificidades locais, como o custo elevado de moradia, que promoveria maior proporção de pessoas não aparentadas em uma mesma residência.

Assim, em razão das limitações impostas pela heterogeneidade regional no Brasil, foi confeccionado o indicador relativo de diversidade. A Tabela
4 - parte B mostra que a correlação entre o indicador bruto e o relativo é positiva $(0,31)$, porém significativo apenas em 14\%. Acredita-se que uma análise conjunta desses dois indicadores permita minimizar algumas das limitações existentes em cada um deles. Para tanto, o índice de diversidade composto foi confeccionado com base nesses dois primeiros. A Tabela 4 - parte B também revela que a correlação entre o índice composto e os demais era positiva, significativa e elevada, respectivamente 0,87 e 0,74 para o índice bruto e o relativo em 2000.

Qual é a relação entre este último indicador e o nível de qualificação em uma RM? A Tabela 4 - Parte A apresenta a correlação entre o índice de diversidade composto e os indicadores de qualificação. Os resultados foram negativos e significativos apenas para a proporção de trabalhadores na economia criativa e para o índice de qualificação superior. Ou seja, os índices de diversidade quando analisados de forma bivariada não apresentam relação positiva com a qualificação, como proposto por Florida (2002a e 2002b), mostrando até tendência contrária, isto é, em uma análise bivariada, as hipóteses de Florida não são corroboradas para o caso brasileiro. Entretanto, medidas assim apresentam muitas 
limitações. Segue um estudo econométrico que complementa o discutido aqui com a inserção de variáveis controle.

\section{Análises econométricas}

Nesta seção, são testadas empiricamente as hipóteses de Florida (2002a e 2002b) com a utilização de modelos econométricos em painel. Como são poucas as RMs no Brasil, foram obtidos dados dos anos 1991 e 2000.

Cada um dos índices de qualificação discutidos acima foi analisado em separado como variável dependente. Como variáveis explicativas, foram incluídas as seguintes: renda domiciliar média (em salários mínimos), grau de urbanização $(\%)$, índice de entretenimento e índice de diversidade composto. As duas primeiras variáveis procuraram controlar alguns dos efeitos espúrios que poderiam existir na relação entre as duas últimas variáveis, que buscam justamente testar as hipóteses propostas por Florida (2002a, 2002b e 2005), e os índices de qualificação. Sociedades mais desenvolvidas tendem a apresentar níveis de qualificação mais elevados e também, de forma geral, mostram maiores níveis de renda, de urbanização e de indivíduos em atividades criativas e de entretenimento (Wojan et al., 2007). Dado que o número de ob- servações é pequeno, foram inicialmente incluídas apenas essas duas variáveis de controle, e optou-se pela utilização de dados em painel. Segue breve explicação sobre esse método. Para uma discussão detalhada, ver Wooldridge (2002).

Sejam $\mathrm{Y} \equiv\left(y_{1}, y_{2}, \ldots, y_{k}\right)$ e $\mathrm{X} \equiv\left(x_{1}\right.$, $\left.x_{2}, \ldots, x_{k}\right)$ variáveis aleatórias observáveis e $\mathrm{C} \equiv\left(c_{1}, c_{2}, \ldots, c_{k}\right)$ variável aleatória não observável, cada uma com $k$ observações. Assumindo o modelo linear com $C$ na forma aditiva, temos:

$y_{i t}=\beta_{0}+x_{i t} \beta+c_{i}+u_{i t}$

onde $i$ se refere a cada uma das RMs, $t=1991$ ou 2000 refere-se aos dois anos estudados, $y_{i t}$ é a variável dependente, que aqui são os índices de qualificação, $\chi_{i t}$ são as variáveis explicativas, e o termo $c_{i}$ trata dos efeitos regionais que não se alteram no período analisado.

Se $\operatorname{cov}\left(x_{i t}, c_{i}\right)=0, c_{i}$ pode ser tratado como variável aleatória, $v_{i t}=c_{i}+u_{i t}$, onde $E\left[v_{i t} \mid x_{i}\right]=0, E\left[u_{i t} \mid x_{i}, c_{i}\right]=0 \quad \mathrm{e}$ $E\left[c_{i} \mid x_{i}\right]=E\left[c_{i}\right]=0$. Temos, então, o modelo de efeitos aleatórios.

Segue uma hipótese desse modelo: $E\left[c_{i}^{2} \mid x_{i}\right]=\sigma_{c}^{2}$. Caso o efeito não observável, representado por $C$, não exista, isto é, se a hipótese nula, $H_{0}: \sigma_{c}^{2}=0$, não for rejeitada, pode-se utilizar um modelo com Mínimos Quadrados Or- 
dinários (MQO) empilhado. Para se testar essa hipótese, utiliza-se o teste de Breush-Pagan.

Em todos os modelos empíricos deste estudo, como é mostrado na Tabela 5 , esse teste rejeitou essa hipótese nula. Ou seja, conclui-se que o modelo clássico de regressão MQO com uma única constante é inapropriado na análise dos dados e que devemos utilizar um modelo em painel, em primeira instância com efeitos aleatórios (Greene, 2003).
Por sua vez, quando $\operatorname{cov}\left(x_{i p} c_{i}\right) \neq 0$, $c_{i}$ não deve ser tratada como variável aleatória, e sim como um parâmetro a ser estimado pelo modelo. Temos, então, o modelo de efeitos fixos.

Os modelos de efeitos fixos tendem a ser mais robustos do que os de efeito aleatório, mas fica a impossibilidade de se incluir variáveis invariantes no tempo, que, no caso deste trabalho, é, por exemplo, a localização geográfica. Para determinarmos a escolha entre os

Tabela 5_Regressões em painel com os dados das regiões metropolitanas

\begin{tabular}{|c|c|c|c|c|c|c|}
\hline \multirow{3}{*}{ Variável independente } & \multicolumn{6}{|c|}{ Variável dependente } \\
\hline & \multirow{2}{*}{$\begin{array}{l}\text { Proporção de } \\
\text { trabalhadores } \\
\text { na economia } \\
\text { criativa }\end{array}$} & \multicolumn{4}{|c|}{ Índice de qualificação } & \multirow[b]{2}{*}{$\begin{array}{c}\text { Indicador } \\
\text { síntese }\end{array}$} \\
\hline & & Superior & $\begin{array}{l}\text { De pós- } \\
\text { graduação }\end{array}$ & $\begin{array}{l}\text { Técnica } \\
\text { superior }\end{array}$ & $\begin{array}{c}\text { Técnica de } \\
\text { pós-graduação }\end{array}$ & \\
\hline Constante & $4,03 * *$ & 0,46 & $-1,82^{* *}$ & $2,52^{*}$ & $-0,083$ & $0,53^{*}$ \\
\hline Renda domiciliar & $1,55^{* *}$ & $1,42^{* *}$ & $0,67 * *$ & $0,96 * *$ & $0,075^{* *}$ & 0,019 \\
\hline Grau de urbanização & 0,00 & 0,01 & 0,00 & 0,00 & 0,00 & 0,00 \\
\hline Índice de entretenimento & $2,89 * *$ & $1,06^{*}$ & $1,74 * *$ & $1,27 *$ & $0,83 * *$ & $0,11 * *$ \\
\hline Índice de diversidade composto & $-0,34$ & 0,13 & 0,04 & 0,24 & $-0,01$ & 0,01 \\
\hline Capital & 0,12 & 1,01 & - & 0,81 & $-0,046 * *$ & - \\
\hline Localização & 0,49 & $1,13^{*}$ & - & $1,45^{*}$ & $-0,00$ & - \\
\hline $\mathrm{R}^{2}$ total & 0,76 & 0,67 & 0,50 & 0,53 & 0,59 & 0,39 \\
\hline Breush-Pagan $(\mathrm{p}<)$ & 0,01 & 0,00 & 0,01 & 0,00 & 0,01 & 0,00 \\
\hline Hausman $(\mathrm{p}<)$ & 0,88 & 0,77 & 0,00 & 0,09 & 0,15 & 0,00 \\
\hline Modelo & Ef. aleatórios & Ef. aleatórios & Ef. fixos & Ef. aleatórios & Ef.aleatórios & Ef. fixos \\
\hline
\end{tabular}


modelos de efeitos aleatórios e os de efeitos fixos, fazemos uso do teste de Hausman, que analisa a diferença entre os coeficientes obtidos nos dois tipos de modelo.

No caso das análises empíricas deste trabalho, como também é mostrado na Tabela 5, esse teste foi significativo para os modelos com o índice de qualificação de pós-graduação e com o índice síntese. Portanto, optou-se por utilizar modelos com efeitos fixos para essas duas variáveis. Por outro lado, os modelos com a proporção de trabalhadores na economia criativa e com o índice de qualificação superior foram não significativos. Definiu-se, então, pelos modelos de efeitos aleatórios. Os outros dois modelos ficaram em uma situação intermediária. Os modelos com os índices de qualificação técnica mostraram probabilidades baixas para o teste de Hausman, porém ambas acima de 5\%. Como descrito em Johnston e Dinardi (1997), mesmo que o modelo de efeitos aleatórios não seja rejeitado pelo teste de Hausman, existem limitações impostas por uma baixa variância nos dados, o que implica a não distinção entre os dois modelos. Ainda assim, decidiu-se por utilizar o modelo com efeitos aleatórios para essas duas variáveis dependentes.
Uma vez obtidos os resultados do teste de Hausman, incluíram-se duas variáveis de localização nos modelos com efeitos aleatórios: uma dummy, indicando se o núcleo da RM era capital de Estado (1 se sim, 0 caso contrário), e outra dummy, indicando se a RM se localiza nas regiões Sul/Sudeste/Centro-Oeste (1 se sim, 0 caso contrário). Procurouse, assim, incluir mais duas variáveis de controle nos modelos que permitiam essa inclusão.

Os resultados obtidos são apresentados na Tabela 5. De forma geral, espera-se que regiões com renda mais elevada apresentem índices de qualificação mais elevados, fato corroborado em todos os modelos, que tiveram coeficientes positivos e significativos para essa variável, com uma única exceção, que foi $\mathrm{o}$ indicador síntese. O coeficiente para o grau de urbanização foi não significativo em todos os modelos, indicando que, uma vez controlados os efeitos das demais variáveis no modelo, essa variável não era correlacionada com os indicadores de qualificação. Os coeficientes para o índice de entretenimento foram todos positivos e significativos, corroborando a primeira das hipóteses de Florida para dados brasileiros. 
Assim, uma vez controlados os efeitos das demais variáveis no modelo, as RMs com maiores índices de entretenimento tendiam também a apresentar maiores níveis de qualificação. $\mathrm{O}$ índice de diversidade composto não mostrou significância estatística em nenhum modelo, sendo assim não corroborada a hipótese de Florida para a relação entre sociedades mais tolerantes e qualificação. As dummies de localização estão presentes em quatro dos modelos. Notase que em dois deles, aqueles com índice de qualificação superior, os coeficientes para a dummy capital foram positivos e significativos e nos demais foram não significativos. Para a dummy de localização regional, os coeficientes foram em sua maioria não significativos e, em um único modelo, para o índice de qualificação técnica de pós-graduação, ele foi negativo e significativo.

\section{6_Estudo com aglomerados}

Aqui as regiões metropolitanas são analisadas com o uso da técnica de aglomerados ou de cluster. Tal técnica permite classificar observações conforme a similaridade entre elas. O objetivo específico desta seção é classificar as áreas estudadas em diferentes grupos relativamente homogêneos, cada qual com caracterís- ticas específicas. Para mais detalhes sobre a técnica, ver Hair et al. (2005). Foram utilizadas as seguintes variáveis na caracterização dos aglomerados: proporção de trabalhadores na economia criativa em 2000, índice síntese em 2000, variação desses dois índices no período e índice de entretenimento em 2000. O índice de diversidade composto não foi inclú́do, uma vez que foi não significativo no estudo econométrico. Os índices de qualificação, por apresentarem correlação positiva, são aqui representados pelo índice síntese.

A Tabela 6 mostra os resultados obtidos para uma análise com cinco aglomerados. As RMs foram classificadas conforme as características de cada grupo, nos quais foram incluídos breves resumos dessas, e a tabela também conta com a composição de cada grupo. Como mostra o primeiro dos aglomerados, três regiões, que aqui são denominadas locais de destaque positivo, Distrito Federal (DF), Florianópolis (SC) e Rio de Janeiro (RJ), tinham valores muito elevados para todos os indicadores, inclusive para o índice de entretenimento. Lembrando que a classificação de valores elevados é relativa às outras regiões do estudo. Os valores das variáveis já eram elevados em 1991, e a variação temporal foi pequena. 


\section{Tabela 6_Análise de aglomerados}

\begin{tabular}{|c|c|}
\hline Característica dos aglomerados & Composição dos aglomerados \\
\hline $\begin{array}{l}\text { Locais de destaque positivo - Indicadores de } \\
\text { qualificação e entretenimento com valores muito elevados e } \\
\text { variação pequena nos indicadores. }\end{array}$ & $\begin{array}{l}\text { Distrito Federal (DF), Florianópolis (SC) e } \\
\text { Rio de Janeiro (RJ) }\end{array}$ \\
\hline $\begin{array}{l}\text { Locais de relativo destaque positivo - Indicadores de } \\
\text { qualificação e entretenimento com valores altos e evolução } \\
\text { média para os indicadores. }\end{array}$ & $\begin{array}{l}\text { Belo Horizonte (MG), Campinas (SP), Curitiba (PR), } \\
\text { Londrina (PR), Maringá (PR), Porto Alegre (RS), } \\
\text { São Paulo (SP), Vale do Itajaí (SC) e Vitória (ES) }\end{array}$ \\
\hline $\begin{array}{l}\text { Locais com qualificação média e evolução positiva - } \\
\text { Indicadores de qualificação e entretenimento com valores } \\
\text { médios e variação positiva. }\end{array}$ & $\begin{array}{l}\text { Baixada Santista (SP), Maceió (AL), Manaus (AM) e } \\
\text { Norte/Nordeste Catarinense (SC) }\end{array}$ \\
\hline $\begin{array}{l}\text { Locais com qualificação média e sociedade vibrante - } \\
\text { Indicadores de qualificação com valores médios e variação } \\
\text { pequena e valores para entretenimento elevado. }\end{array}$ & $\begin{array}{l}\text { Belém (PA), Fortaleza (CE), Goiânia (GO), Natal (RN), } \\
\text { Recife (PE), Salvador (BA) e São Luís (MA) }\end{array}$ \\
\hline $\begin{array}{l}\text { Locais com qualificação inferior e evolução positiva - } \\
\text { Indicadores de qualificação e entretenimento com valores } \\
\text { baixos e variação positiva para muitos deles. }\end{array}$ & Vale do Aço (MG) \\
\hline
\end{tabular}

Fonte: IBGE. Censos Demográficos (1991 e 2000). Elaboração própria.

Um segundo grupo de RMs foi denominado como locais de relativo destaque positivo. As variáveis de qualificação e de entretenimento em 2000 tinham valores relativamente altos, e a variação foi média. As RMs de Belo Horizonte (MG), Campinas (SP), Curitiba (PR), Londrina (PR), Maringá (PR), Porto Alegre (RS), São Paulo (SP), Vale do Itajaí (SC) e Vitória (ES) tinham essas características.

Um terceiro aglomerado contava com as RMs Baixada Santista (SP), Maceió (AL), Manaus (AM) e Norte/
Nordeste Catarinense (SC). Essas regiões apresentavam valores médios para as variáveis de qualificação e entretenimento, bem como variação positiva nos indicadores, sendo assim denominadas locais com qualificação média com evolução positiva.

O quarto aglomerado contava com as seguintes RMs: Belém (PA), Fortaleza (CE), Goiânia (GO), Natal (RN), Recife (PE), Salvador (BA) e São Luís (MA). Essas regiões tinham valores de qualificação semelhantes ao anterior, mas com 
evolução pequena; porém, contavam com indicadores de entretenimento elevado. Foram assim denominadas como locais com qualificação média e sociedade vibrante. Por fim, uma única RM, Vale do Aço (MG), formava o quinto aglomerado, com valores mais baixos para todas as variáveis, mas com variação positiva no período.

\section{7_Conclusão}

O ponto de partida deste texto é a relação entre a aglomeração de capital humano e o desenvolvimento socioeconômico de cidades e regiões. Tendo como base teórica o proposto por Florida (2002a, 2002b e 2005), foi feita uma análise empírica sobre a distribuição de indivíduos qualificados nas regiões metropolitanas do Brasil (RMs), com a utilização de diversos indicadores. Os resultados mostraram que algumas RMs se destacavam positivamente, como Florianópolis (SC) e o Rio de Janeiro (RJ), enquanto outras apresentavam valores menos marcantes, como o Vale do Aço (MG).

Em seguida, foram analisadas empiricamente duas das hipóteses de Florida (2002a, 2002b e 2005) para dados brasileiros. A primeira delas sugere que a existência de uma sociedade local vibran- te cria uma atmosfera favorável à atração de indivíduos talentosos e criativos, que por sua vez promovem o desenvolvimento regional. A segunda hipótese é que a diversidade populacional existente em uma localidade reflete maior tolerância na absorção de indivíduos de diferentes tipos e também torna o local mais atraente para indivíduos qualificados.

Foram utilizados dados em painel com modelos com efeitos aleatórios e outros com efeitos fixos, dependendo do índice de qualificação analisado. Verificou-se que parte do proposto por esse autor foi empiricamente apreendido para o Brasil. RMs com índices de entretenimento, proxi para a primeira hipótese, mais elevados tendiam a apresentar maior proporção de indivíduos qualificados. Entretanto, esse fato não ocorreu com os índices de diversidade populacional, proxi para a segunda hipótese, em que os resultados foram não significativos.

Por fim, foram determinados diferentes perfis para as RMs com a utilização da técnica de aglomerados, de acordo com a qualificação local e características relacionadas ao entretenimento. Três regiões metropolitanas - Distrito Federal (DF), Florianópolis (SC) e Rio de Janeiro (RJ) - sobressaem-se positi- 
vamente com relação a todos os indicadores e são locais naturalmente atraentes para indivíduos qualificados. Outras nove RMs - Belo Horizonte (MG), Campinas (SP), Curitiba (PR), Londrina (PR), Maringá (PR), Porto Alegre (RS), São Paulo (SP), Vale do Itajaí (SC) e Vitória (ES) -, todas localizadas nas regiões Sul/ Sudeste do país, também apresentavam variáveis com níveis relativamente elevados. As demais 12 RMs tinham valores menos elevados para qualificação, quase todas do Norte/Nordeste do Brasil, áreas de menor qualificação média, ou são centros urbanos de tamanho médio do interior do Sul/Sudeste do país. Todas essas regiões, apesar de terem níveis de qualificação relativamente inferiores quando comparadas com as demais RMs, mostraram evolução positiva nos níveis de qualificação.

Toda a análise sugere uma evolução positiva do conjunto das RMs, com uma diminuição na variabilidade dos níveis de qualificação entre as regiões no período analisado. 


\section{Referência bibliográficas}

ACEMOGLU, D. A

microfundation for social

increasing returns in human capital. Quarterly Journal of Economics, v. 111, n. 3, p. 779-804, 1996.

\section{AUDRETSCH, D.; FELDMAN,}

M. R \& D spillovers and the geography of innovation and production. American Economic Review, v. 86, n. 3, p. 630-640, 1996.

\section{BOSCHMA, R.; FRITSCH, M.}

Creative class and regional growth in Europe. In: JACUBOWSKA, P.; KUKLINSKI, A.; ZUBER, P. (Eds.). The future of European Regions. Varsóvia: Ministério do Desenvolvimento Regional, 2007.

CLARK, G.; GERTLER, M.; FELDMAN, M. Economic geography: transition and growth. In: CLARK, Gordon; GERTLER, Meric; FELDMAN, Maryann (Eds.). The Oxford Handbook of Economic Geography. Oxford: Oxford University Press, 2000. p. 3-17.

DE HAAN, A. Livelihoods and poverty: The role of migration A critical review of the migration literature. The Journal of Development Studies, v. 36, n. 2, p. 1-47, 1999.
EVANS, A. The assumption of equilibrium in the analysis of migration and interregional differences: A review of some recent research. Journal of Regional Science, v. 30, n. 4, p. 515-531, 1990.

FALCK, O.; FRITSCH, M.; HEBLICH, S. Bohemians, human capital and regional economic growth. CESifo Working Paper Series, n. $2715,2009$.

FELDMAN, M. Location and innovation: the new economic geography of innovation, spillovers, and agglomeration. In: CLARK, Gordon; GERTLER, Meric; FELDMAN, Maryann (Eds.). The Oxford Handbook of Economic Geography. Oxford: Oxford University Press, 2000. p. 373-394.

FLORIDA, R. The economic geography of talent. Annals of the Association of American Geographers, v. 92, n. 4, p. 743-755, 2002a.

FLORIDA, R. Bohemia and economic geography. Journal of Economic Geography, n. 2, p. 55-71, 2002b.

FLORIDA, R. Cities and the creative class. New York, London: Routledge, 2005.
FLORIDA, R; TINAGLI, I. Europe in the creative age. 2004. Disponível em: http:// creativeclass.org/acrobat/Europe in_the_Creative_Age_2004.pdf. Acesso em: 15 maio 2007.

FLORIDA, R.; MELLANDER, C.; STOLARICK, K. Inside the black box of regional development: buman capital, the creative class and tolerance. Royal Institute of Technology, 2007. (Cesis Working paper series, n. 88).

\section{GLAESER, E. The new} economics of urban and regional growth. In: CLARK, Gordon; GERTLER, Meric; FELDMAN, Maryann (Eds.). The Oxford Handbook of Economic Geography. Oxford. Oxford University Press, 2000. p. 83-98.

GLAESER, E.; SCHEINKMAN, J.; SHELEIFER, A. Economic growth in a cross-section of cities. Journal of Monetary Economics, v. 36, n. 1, p. 117-143, 1995.

GLAESER, E.; KOLKO, J.; SAIZ, A. Consumer city. Journal of Economic Geography, v. 1, n. 1, p. 27-50, 2001.
GOLGHER, A. As cidades e a classe criativa no Brasil: diferenças espaciais na distribuição de indivíduos qualificados nos municípios brasileiros. Revista Brasileira de Estudos de População, v. 25, n. 1, p. 109-129, 2008.

GRAVES, P.; MUESER, P. The role of equilibrium and disequilibrium in modeling regional growth and decline: A critical reassessment. Journal of Regional Science, v. 33, n. 1, p. 69-84, 1993.

GREENE, W. Econometric Analysis. 5. ed. Prentice Hall, Nova Jersey, 2003.

\section{GREENWOOD, M. Human} migration: Theory, models, and empirical studies. Journal of Regional Science, v. 25, n. 4, p. 521-544, 1985. HAIR J.; ANDERSON, R.; TATHAM, R.; BLACK, W. Análise multivariada de dados. 5. ed. Brasil: Bookman, 2005.

HARRIGAN, F.; MCGREGOR, P. Equilibrium and disequilibrium perspectives on regional labor migration. Journal of Regional Science, v. 33, n. 1, p. 49-67, 1993.

IBGE. Censo Demográfico. Brasil: IBGE, 1991.

IBGE. Censo Demográfico. Brasil: IBGE, 2000. 
JACOBS, J. Morte e vida das grandes cidades. 2. ed. Brasil: Martins

Fontes, 2001.

JOHNSTON, J.; DINARDI, J. Econometric methods. 4. ed. The

McGraw-Hill Companies, Nova York, 1997.

KANBUR, R.; RAPOPORT, H. Migration selectivity and the evolution of spatial inequality. Journal of Economic Geography, v. 5, n. 1, p. 43-57, 2005.

KNAPP, T.; GRAVES, P. On the role of amenities in models of migration and regional development. Journal of Regional Science, v. 29, n. 1, p. 71-87, 1989.

LEE, S.; FLORIDA, R.; ACS, Z. Creativity and entrepreneurship: A regional analysis of new firm formation. Regional Studies, v. 38, n. 8, p. 879-891, 2004.

LUCAS, R. On the mechanics of economic development. Journal of Monetary Economics, v. 22, n. 1, p. 3-42, 1988.

MATHUR, V. Human capitalbased strategy for regional economic development. Economic Development Quarterly, v. 13, n. 3, p. 203-216, 1999.

MELLANDER, C.; FLORIDA,

$\mathrm{R}$. The creative class or buman capital - explaining regional development in Sweden. Royal Institute of Technology, 2007. (Cesis Working Paper, n. 79).
MORETTI, E. Estimating the social return to education: Evidence from longitudinal and repeated cross-section data. Journal of Econometrics, n. 121, p. 175-212, 2004.

NEARY, J. Of hype and hyperbolas: Introducing the new economic geography. Journal of Economic Literature, n. 39, p. 536-561, 2001.

PORREL, F. Intermetropolitan migration and quality of life. Journal of Regional Science, v. 22, n. 2, p. 137-158, 1982.

SCHACHTER, J.; ALTHAUS,

P. The assumption of equilibrium in models of migration. Journal of Regional Science, v. 33, n. 1, p. 85-88, 1993.

SCOTT, A. A perspective of economic geography. Journal of Economic Geography, v. 4, n. 5, p. 479-499, 2004.

SIMON, C. Human capital and metropolitan employment growth. Journal of Urban Economics, v. 43, n. 2, p. 223-243, 1998.

STILLWELL, J.; CONGDON, P. Migration modeling: Concepts and contents. In: STILLWELL, J.; CONGDON, P. (Ed.). Migration Models: Macro and micro approaches, edited by J. Stillwell e P. Congdon. New York: Belhaven Press, 1991. p.1-16.
WESTLUND, H.; CALDONILUNDBERG, F. The creative class and social capital - civil society, regional development and hightech employment in Japan. Royal Institute of Technology, 2007. (Cesis Working Paper, n. 112).

WOJAN, T.; LAMBERT, D.; MCGRANAHAN, D. Emoting with their feet: Bohemian attraction to creative milieu. Journal of Economic Geography, v. 7, n. 6, p. 711-736, 2007.

WOOLDRIDGE, J. Econometric analysis of cross section and panel data. Cambridge, MA: MIT Press, 2002.

E-mail de contato do autor:
$\vdots$
$\vdots$

agolgher@cedeplar.ufmg.br

Artigo recebido em maio de 2009;
aprovado em maio de 2010.

